\section{Caracterización del tratamiento de la toxoplasmosis gestacional}

\section{Characterization of the treatment of gestational toxoplasmosis \\ Caracterização do tratamento da toxoplasmose gestacional}

Iris Victoria Guedez Rojas

vickyvictoria.1@hotmail.com

Luís Alfonzo Barroso Oria

luisbarroso_47@hotmail.com

Recibido diciembre 2019 / Revisión enero 2020 / Aceptado 1 de mayo 2020

\section{RESUMEN}

En el presente trabajo, se delimitó como objetivo: Caracterizar el tratamiento de las pacientes con diagnóstico inicial de toxoplasmosis gestacional atendidas en el hospital "Dr. José Gregorio Hernández" en el periodo de enero 2018 hasta junio 2018. Materiales y Métodos: se diseñó una investigación de tipo descriptiva, observacional, y transversal. La muestra estuvo comprendida por 67 pacientes con serologías positivas de inmunoglobulina G o M a las que se les aplicó un instrumento de recolección de datos en el que se determinó la frecuencia de diagnósticos por seroconversión de IGG e IGM, los medicamentos más utilizados en los casos positivos, y se caracterizó el posterior seguimiento de la afección. Resultados: De 67 pacientes inicialmente diagnosticadas como Toxoplasmosis Gestacional, solo trece de ellas (19,4\%) tuvo seroconversión de anticuerpos durante el embarazo. A la totalidad de la población se le indico algún tipo de tratamiento, el más frecuente fue Azitromicina (58\%), El $100 \%$ de los casos fueron seguidos por ecografía, y el (95\%) de ellos valorado por algún especialista.

Palabras clave: Toxoplasmosis; diagnóstico, serología

\begin{abstract}
In the present work, the objective was delimited: To characterize the treatment of patients with an initial diagnosis of gestational toxoplasmosis treated at the "Dr. José Gregorio Hernández" in the period from January 2018 to June 2018. Materials and Methods: a descriptive, observational, and crosssectional research was designed. The sample was comprised of 67 patients with positive immunoglobulin $\mathrm{G}$ or $\mathrm{M}$ serologies to which a data collection instrument was applied in which the frequency of diagnoses due to seroconversion of IGG and IGM was determined, the drugs most used in cases positive, and the subsequent follow-up of the condition was characterized. Results: Of 67 patients initially diagnosed as Gestational Toxoplasmosis, only thirteen of them (19.4\%) had antibody seroconversion during pregnancy. Some type of treatment was indicated to the entire population, the most frequent was Azithromycin (58\%), 100\% of the cases were followed by ultrasound, and (95\%) of them were evaluated by a specialist.
\end{abstract}

Key words: Toxoplasmosis; diagnosis, serology
IG: Hospital Materno Infantil Dr. José Gregorio Hernández. Acarigua. Especialista adjunto en Ginecología y Obstetricia.

LB: Universidad Politécnica Territorial del estado Portuguesa. Especialista en Epidemiologia, Venezuela. 
IG: Hospital Materno Infantil Dr. José Gregorio Hernández. Acarigua. Especialista adjunto en Ginecología y Obstetricia.

LB: Universidad Politécnica Territorial del estado Portuguesa. Especialista en Epidemiologia, Venezuela.

\section{RESUMO}

O presente trabalho, delimitou-se o objetivo: caracterizar o tratamento de pacientes com diagnóstico inicial de toxoplasmose gestacional atendidos no centro de saúde "José Gregorio Hernández" no período de janeiro de 2018 a junho de 2018. Materiais e Métodos: pesquisa descritiva, observacional e transversal. A amostra foi composta por 67 pacientes com testes de sorologias positivas para imunoglobulina $\mathrm{G}$ ou $\mathrm{M}$, nas quais foi aplicado um instrumento de coleta de dados. Foi possível determinar a frequência dos diagnósticos por soro-conversão de IGG e IGM, os medicamentos mais utilizados nos casos positivos e o seguimento subsequente da condição foi caracterizado. Resultados: Dos 67 pacientes inicialmente diagnosticados como Toxoplasmose Gestacional, apenas treze deles (19,4\%) apresentaram soroconversão de anticorpos durante a gravidez. Foi indicado algum tipo de tratamento para toda a população, sendo a azitromicina o medicamento mais frequentemente indicado para os pacientes (58\%). $100 \%$ dos casos foram seguidos por ultrassonografia e (95\%) deles foram avaliados pelo especialista.

Palabras clave: Toxoplasmose; diagnóstico; sorologia

\section{INTRODUCCIÓN}

$\mathrm{E}$ l toxoplasma gondii es el agente responsable de la toxoplasmosis, una de las infecciones parasitarias más frecuentes en el mundo. La infección en el hombre es habitualmente asintomática o puede tener un curso clínico benigno. Sin embargo la infección congénita 0 en los pacientes inmunodeprimidos puede ser muy seria y mortal (1).

Las infecciones antes del embarazo producen inmunidad duradera. Cuando la infección se adquiere por primera vez durante el embarazo esta puede traer consecuencias graves para el feto, como hidrocefalia, microcefalia, calcificaciones cerebrales, coriorretinitis, o en el peor de los casos terminar en un aborto, dependiendo de la fecha en que la madre se infectó. Por esto, es importante detectar las infecciones recientes en el curso del control prenatal. (2)

En Venezuela la Toxoplasmosis congénita es un problema de salud pública importante y esta infección es de muy alta prevalencia, por lo tanto se considera que la mitad de la población en Venezuela ha tenido contacto con este parásito (3).
Según estudios realizados en diferentes regiones, cada año aparecen de 2 a10 casos con toxoplasmosis congénita por cada 1.000 recién nacidos $(2,4,5)$.

Según el estudio nacional de salud realizado en 1987 en 14 estados de Venezuela la distribución de 825 pacientes estudiados fue la siguiente: Lara 776; Portuguesa 17; Yaracuy 15; Falcón 6; Aragua 2, y Trujillo, Carabobo, Barinas, Mérida, Apure, Sucre, Táchira, Zulia y Monagas uno cada uno. De 380 mujeres que consultaron por síntomas y signos obstétricos, $205(54,0 \%)$ fueron positivas en la serología; de estas, nueve embarazadas, una con amenaza de aborto, seis con abortos, tres con mortinatos y una con parto prematuro presentaron títulos serológicos de 1: 16 a 384 o más altos (6).

La prevalencia de la toxoplasmosis en la mayoría de los países se detecta serológicamente, y está en un rango entre 40 y 50\% (7). Las características del medio ambiente influyen en la prevalencia, pues esta es mayor en regiones calientes y húmedas, pero más baja en climas secos y fríos. Los factores culturales tienen relación especial con este parásito, pues la costumbre de comer carne cruda o mal 
cocida y la de tener gatos en las casa favorecen la infección.

La toxoplasmosis es una de las zoonosis parasitarias más difundidas en el mundo. La Organización Mundial de la Salud estima que más de un tercio de la población mundial está infectada (7). Es una infección autolimitada, de muy bajo riesgo en las personas inmunocompetentes. En condiciones normales ocurre una vez en la vida y deja un estado de inmunidad humoral y celular permanente. Ello obliga a distinguir entre la infección muy común y la enfermedad infrecuente. Se observa en ambos sexos en la misma proporción. La infección puede ser intrauterina o adquirida posnatal.

En Venezuela según el Instituto de Medicina Tropical (3). en un estudio publicado durante 2016 con una muestra de 68. 622 pacientes se determinó una seroprevalencia de $50.9 \%$, lo cual revela la magnitud de la infección como problema de Salud Pública. Las embarazadas no escapan a esta realidad, podría estimarse que un número importante son desapercibidas en las consultas del Sistema Público Nacional de Salud.

La infección aguda en la embarazada, por lo general es asintomática y sólo puede ser detectada con pruebas serológicas. La transmisión del parásito de la madre al hijo únicamente puede ocurrir cuando la infección se adquiere por primera vez durante el embarazo y aumenta gradualmente con el progreso de la gestación.

Según el Consenso Argentino de Toxoplasmosis Congénita (8) en términos generales, un tercio de las madres con infección aguda darán a luz un hijo con toxoplasmosis, en su mayoría con un desarrollo normal; sin embargo, el 4\% tiene posibilidades de morir, tener un daño neurológico permanente o compromiso visual desde los primeros años de vida.
En el municipio Palavecino del estado Lara, Travieso en el 2014 (9), determinó una seroprevalencia en gestantes del $38 \%$, constituyendo una frecuencia alta para ese grupo poblacional, sin embargo no se disponen de caracterizaciones acerca del manejo común de la Toxoplasmosis gestacional en Venezuela y esto refleja la necesidad de describir el problema en función de generar estrategias de salud pública que normen los procedimientos a realizar en cuanto al diagnóstico $y$ tratamiento eficaz.

Según Epidemiologia Ambiental Portuguesa (10), existe una tendencia cada vez más frecuente en los reportes de algunos laboratorios de la localidad, que solo se resumen en "Test para toxoplasmosis positivo o negativo", sin precisar el o los anticuerpos que se determinó en cada caso, la concentración de estas inmunoglobulinas en suero materno y la técnica de laboratorio que se utilizó para tal determinación. De igual manera, a pesar de lo impreciso de estos resultados, se decide en algunos de estos casos diagnosticar o no diagnosticar $\mathrm{y}$ tratar o no tratar a la paciente. Ante los elementos descritos es necesario preguntarse ¿Se estará suministrando tratamiento a todas aquellas pacientes que lo requieran, y se estará medicando a aquellas que no lo ameriten durante el periodo de estudio?

La toxoplasmosis gestacional a nivel mundial es un problema de Salud Pública debido a su elevada incidencia, según OMS (7), se calculó que la incidencia anual de la toxoplasmosis congénita a nivel mundial es de 190100 casos. En Venezuela no figura dentro de las enfermedades notificables en los formatos de vigilancia epidemiológica del Ministerio del poder popular para la salud, a pesar de la existencia de diversos estudios que caracterizan la seroprevalencia en distintas regiones. 
El objetivo principal de la investigación fue caracterizar el tratamiento de la toxoplasmosis gestacional en la consulta prenatal del Hospital Materno Infantil Dr. José Gregorio Hernández en un periodo comprendido de enero 2018 a junio 2018. Para ello, se describirá la frecuencia de diagnósticos serológicos de Toxoplasmosis Gestacional, también se identificará los distintos fármacos que fueron prescritos a las pacientes diagnosticadas en el periodo de estudio, para detallar el seguimiento obstétrico posterior desde el punto de vista de sus interconsultas y hallazgos ecográficos.

Caracterizar el tratamiento de las pacientes diagnosticadas con toxoplasmosis, permitiría revisar a la luz de una investigación, la situación actual del uso de diversos fármacos que al utilizarse de manera incorrecta, sin ajuste a protocolos, podrían causar daños en el binomio materno fetal: a su vez, está investigación, constituirá un punto de partida para subsecuentes estudios en el estado Portuguesa acerca del tema en cuestión.

No se pudo determinar la existencia de investigaciones publicadas que caractericen el manejo obstétrico de esta afección en Venezuela, allí radica la importancia de este estudio, porque conforma un hecho inédito en el estado, particularmente en el Hospital Materno Infantil Dr. José Gregorio Hernández, el cual constituye el núcleo central de esta investigación.

La prevalencia de anticuerpos antiToxoplasma gondii en las gestantes determina el porcentaje de pacientes protegidas de una reinfección dentro de una población; en cambio aquellas seronegativas, al no haber entrado en contacto con el parásito son susceptibles de adquirir la infección durante la gestación, aumentando exponencialmente la posibilidad de transmitir el parasito al feto. ¿Se estará diagnosticando de manera apropiada y diferenciada estas dos condiciones distintas? $i$ El tratamiento en cada caso está llevado a cabo de manera diferenciada? el objetivo fundamental del presente trabajo ha sido caracterizar el tratamiento integral de esta infección durante el embarazo en la consulta prenatal del Hospital Materno Infantil Dr. José Gregorio Hernández de la ciudad de Acarigua.

\section{MATERIALES Y MÉTODOS}

$\mathrm{E}$ $\mathrm{n}$ el desarrollo de este estudio se realizó una investigación de tipo observacional, transversal $\mathrm{y}$ descriptiva. La población estuvo constituida por 1122 pacientes que acudieron al centro asistencial durante el periodo de estudio. Para la muestra, se utilizó un muestreo intencional no probabilístico en 67 pacientes que cumplieron con los criterios de inclusión los cuales se detallan a continuación.

\section{Criterios de inclusión}

Pacientes en las que se haya indicado algún fármaco para el tratamiento de la Toxoplasmosis gestacional en el momento del embarazo y que acudan a la consulta prenatal o hayan estado ingresadas durante el periodo de estudio.

Pacientes con serologías Positivas IGM o IGG realizadas por técnicas de Inhibición de la hemoaglutinación y Eliza y en las que se haya diagnosticado toxoplasmosis gestacional.

Pacientes en las que se detecte seroconversión de anticuerpos IGG en dos tomas subsecuentes en un espacio de 3 semanas.

Pacientes con serologías negativas en las que se haya detectado elevación de IGG durante su embarazo. 


\section{Criterios de exclusión}

Mujeres que previa información suministrada por la investigadora no acepten participar voluntariamente en el estudio.

\section{RESULTADOS Y DISCUSIÓN}

$\mathrm{U}$ na vez recolectada la información referente a la caracterización del tratamiento de la toxoplasmosis gestacional en el Hospital Materno Infantil "Dr. José Gregorio Hernández" de Acarigua, estado Portuguesa durante el periodo enero 2018 hasta junio 2018, mediante la aplicación de un instrumento diseñado para tal fin, se procede al análisis e interpretación de la misma a través de tablas y gráficos de distribución en frecuencia y porcentajes.

El total de la muestra fue de 67 pacientes; 48 de ellas (71\%) tenía Serología IGM cuantitativa positiva indistintamente del método empleado. 36 de ellas (53\%) estaba positiva con la serología IGG. En 21 de ellas (31\%) la IGM e IGG estaban positivas. En ninguna paciente se pudo constatar la indicación ni la realización del Test de Avidez. Solo el 19 $\%$ de los casos (13 pacientes) tuvo seroconversión de IGG en presencia de IGM positiva.

En el 100 por ciento de las pacientes se utilizó algún tipo de fármaco. El $38 \%$ de las pacientes (26 de ellas) les fue indicado el uso de la Espiramicina durante un mes. 2 pacientes $(2.9 \%)$ fueron tratadas con pirimetamina más ácido fólico hasta el final del embarazo, a 39 pacientes (58\%) les fue indicada la azitromicina como monodosis durante cinco días.

En el caso de las 13 pacientes con seroconversión (19\%) el tratamiento fue distribuido de la siguiente forma: 8 pacientes con Azitromicina por 5 días (62\%) 3 pacientes con Provamicina (23\%) por 30 días, 2 pacientes con Pirimetamina
+ Ácido fólico (15\%) hasta el final del embarazo.

El $100 \%$ de las pacientes se realizó por lo menos 2 ecografías durante el embarazo con resultados dentro de los límites normales. El 95 \% (64 de ellas) fue atendida por algún obstetra, el $64 \%$ fue referida a Epidemiologia y ninguna de ellas fue referida a Infectología

\section{Discusión}

En España, González et al (11) a través de un estudio retrospectivo descriptivo en el que se propusieron conocer los resultados perinatales obtenidos en las pacientes con seroconversión para toxoplasma durante la gestación con una muestra de 139 gestantes, de las cuales un $85 \%$ presentó la seroconversión para toxoplasma en el primer trimestre, el 11\% en el segundo y el $4 \%$ restante en el tercer trimestre. Se realizó un análisis en tres grupos según el resultado de la avidez de la inmunoglobina G: débil (32\%), intermedia (17\%) y fuerte (50\%). En el caso de la presente investigación la frecuencia de aparición de seroconversión fue de $19 \%$, en un total de 67 pacientes estudiadas, en ningún caso se determinó el test de Avidez IGG, sino se tomó como referencia la seroconversión de la IGG al triple del valor inicial en presencia de IGM positiva, ambos trabajos coinciden en la utilización de diagnóstico serológico como elemento de valor en la toma de decisiones de la Toxoplasmosis gestacional, de tal forma que tanto frecuencia como prevalencia de casos se mantienen en el rango del segundo quartil del total de la muestra,

En el caso del ya citado trabajo de Christiana $F$ et al, (12) en el Hospital universitario de Lusaka, Zambia, en un diseño transversal, descriptivo, cuyo objetivo principal fue caracterizar la seroconversión por IGG test de Elisa en presencia de IGM positivo, en una 
población de 411 mujeres embarazadas, se demostró que la incidencia de esta infección en el embarazo fue de 0,5 \% con solo 3 casos de seroconversión las cuales fueron tratadas con Espiramicina cada 8 horas durante todo el embarazo obteniendo al año de seguimiento una incidencia negativa de toxoplasmosis congénita. Hay que tomar en cuenta que en el diseño de esta investigación la propuesta fue determinar incidencia, en el caso de la actual investigación se estimó la frecuencia de seroconversión en un grupo de pacientes con serología positivas, al mismo tiempo es necesario puntualizar que en la presente investigación el fármaco más utilizado fue la azitromicina como monodosis durante cinco días (58\%).

Por su parte Cuellar y Molina (13) en caracterización de la Toxoplasmosis gestacional en una clínica privada de Neiva (Colombia), en 2014. Los principales resultados arrojaron que la prevalencia de toxoplasmosis gestacional fue del 3\%. La edad gestacional promedio en el que se realizó el diagnóstico fue de 23 semanas. El promedio de seroconversión fue del 34\%, El tratamiento farmacológico más utilizado fue la espiramicina. El presente trabajo coincide con la frecuencia de aparición de Toxoplasmosis gestacional inferior al 40 $\%$, es decir 2do. Cuartil, (19 \%) diagnosticadas por seroconversión. Y es diferente en cuanto a la frecuencia de utilización de fármacos, siendo en este caso la Azitromicina como la de uso más frecuente sin tomar en cuenta el diagnostico de Seroconversión.

En la investigación de Aguayo (14) titulada Prevalencia de Toxoplasmosis y Factores de Riesgo asociados en embarazadas que acuden al primer control prenatal en el Centro de Salud de Quero, Provincia Tungurahua en 2013 en Ecuador obtuvo como resultado que la prevalencia de toxoplasmosis fue del $27 \%$ en embarazadas que acuden al primer control prenatal. En la investigación actual no se determinó prevalencia sino frecuencia de casos de aparición sobre una muestra intencional no probalistica, en ese sentido el resultado fue de $19 \%$ de casos de sero conversión sobre el total de la muestra. El fármaco más utilizado en la investigación de Aguayo fue la espiramicina en contraste con la azitromicina en el presente estudio.

En el estudio descriptivo realizado en la ciudad de Maracay encabezado por Fernández (15) titulado Seroepidemiología de Toxoplasmosis en habitantes del estado Aragua. Se evidenció que $49,9 \%$ de los participantes $\quad(181 / 363)$ tenían anticuerpos totales anti-T. gondii. Por otra parte, no se observaron diferencias estadísticas $(p>0.05)$ entre grupos etarios, género y seroprevalencia. En este punto es importante resaltar que en la búsqueda de distintas investigaciones sobre el tratamiento de la toxoplasmosis gestacional, partiendo del hecho del diagnóstico serológico adecuado son verdaderamente escazas, permaneciendo la mayoría de ellas inéditas, la mayor parte de las investigaciones disponibles en versiones publicadas en revistas indexadas se refieren a la prevalencia de algún suero específico sin hacer énfasis en las pacientes que deben tratarse y la que no deben tratarse, con poca diferenciación entre la toxoplasmosis gestacional aguda y la toxoplasmosis crónica gestacional en la paciente inmunocompetente.

\section{CONCLUSIONES}

$\mathrm{S}$ e observó que el $100 \%$ de las pacientes eran diagnosticadas y tratadas sin que se cumpliera el criterio de Seroconversión. De una población de 67 pacientes solo 13 (19\%) cumplieron dicho criterio. 48 pacientes(71\%) tuvieron Serología IGM cuantitativa positiva indistintamente del método empleado. 36 de ellas (53\%) 
estaba positiva con la serología IGG. En 21 de ellas (31\%) la IGM e IGG estaban positivas. En ninguna paciente se pudo constatar la indicación ni la realización del Test de Avidez.

Se identificó la indicación de espiramicina como monodosis por 21 días, la pirimetamina hasta el final del embarazo y la azitromicina. El fármaco prescrito con más frecuencia fue la Azitromicina $(500$ $\mathrm{mg}$ ) diarios por 5 días, el mismo se utilizó en un $58 \%$ de ellas (39 pacientes) y en el grupo que mostro seroconversión en un 61 $\%$ (8 pacientes)

El $95 \%$ del total de las pacientes fueron evaluadas por algún obstetra en el transcurso de su embarazo, $64 \%$ fue interconsultada con epidemiología y ninguna de ellas con infectología, en el $100 \%$ de los casos se realizó por lo menos 2 ecografías en el transcurso del embarazo

- Conflicto de intereses. Ninguno declarado por los autores.

- Financiación. Ninguna declarada por los autores

- Agradecimiento: Ninguno manifestado por los autores

- Investigación realizada considerando los tratados bioéticos

\section{REFERENCIAS}

1.- Gómez JE, Diagnóstico de la toxoplasmosis humana: nuevos conceptos y técnicas. Revista Medicina y Laboratorio [internet] 2000; 9: 3-4[fecha de acceso 10 de noviembre 2017]; Disponible en: http://www.scielo.org.co/pdf/rsap/v4n3/ v4n3a06.pdf

2.-Gómez JE, Castaño JC, Montoya MT. Toxoplasmosis congénita en Colombia: un problema subestimado de salud pública. Colombia médica [internet] 1995; 26: 6670. [Fecha de acceso 1 de noviembre 2017]; Disponible en: www.scielo.org.co/pdf/rsap/v4s1/v4s1a0 3.pdf
3.- Luciano M, Zoraida D, M Aponte, A Muñoz-Calderón, Alarcón Belkisyolé. Fifteen Years of Toxoplasmosis Screening at the Institute of Tropical Medicine, a Diagnostic Reference Center in Venezuela. Libertas Académica. [Internet] 2016 p.1 [fecha de acceso 10 de septiembre 2017]; Disponible en:

https://www.researchgate.net/publication/ 291424101_Fifteen_Years_of_Toxoplasmosis _Screening_at_the_Institute_of_Tropical_Medi cine_a_Diagnostic_Reference_Center_in_Vene zuela

4.- Gómez JE, Montoya MT, Castaño JC, Ríos MP, Montoya MT. Toxoplasmosis congénita e hidrancefalia. Acta Med Col 1992; [internet] 17: 457-458. [Fecha de acceso 8 de agosto 2017]; Disponible en http://www.scielo.org.co/pdf/rcog/v61n3 /v61n3a12.pdf

5.-Gómez JE, Montoya Mt, castaño JC, Ríos MP, Pérez JC. Epidemiología de la infección por Toxoplasma gondii en gestantes de Armenia (Quindio). Colombia Méd [internet] 1993; 24: 14-18. [Fecha de acceso 15 de noviembre 2017]; Disponible en:

http://revistas.unicesar.edu.co/index.php /microtrop/article/view/45

6.-Bonfante-Garrido R. Álvares N., De Anzola N, De Crespo L, Cabarcas .R y De Peñaloza S. Toxoplasmosis en 14 estados de Venezuela. Bol of Sanzi t'anam [internet] 96, 1987. [Fecha de acceso 15 de noviembre 2017]; Disponible en: http://www.scielo.org.ve/scielo.php?scrip $\mathrm{t}=$ sci_arttext\&pid=S131525562009000100010

7.-Organización Mundial de la Salud. Boletín Epidemiológico 2016. Número 7, [internet] julio 2016, 465-544[fecha de acceso 10 de enero 2018]; Disponible en: http://www.who.int/bulletin/volumes/91 /7/14-111732-ab/es/).

8.- Consenso Argentino de Toxoplasmosis. Ministerio de Salud Argentina. Rev. Argentina [internet] 2014. [Fecha de acceso 10 de diciembre 2017]; Disponible en:

http://www.scielo.org.ar/scielo.php?script =sci_arttext\&pid=S0025-

76802008000100013

9.- Travieso L., Triolo M. Seroprevalencia de anticuerpos contra Toxoplasma gondii 
en gestantes del municipio Palavecino, estado Lara, Venezuela. Kasmera.

[Internet] 13 enero- junio 2014. [Fecha de acceso 15 de octubre 2017]; Disponible en: http://www.scielo.org.ve/scielo.php?scrip $\mathrm{t}=$ sci_arttext\&pid=S0075-

52222006000100002

10.- Barroso L. Toxoplasmosis Gestacional. La Cascabel y el Gato. Conferencia en Dirección de Salud del estado Portuguesa; Acta Dirección de Salud Ambiental VII, Sep. 13 Folio IV. Acarigua, Portuguesa. 2016

11.- Rodríguez L., Cernadas S, Cerviño E, González R y López Ramón y Cajal C. Resultados perinatales y manejo de las pacientes con seroconversión para toxoplasma durante la gestación. España. Prog. Obstet. Ginecol. [Internet] 2016; 59[fecha de acceso 1 de abril 2018]; Disponible en: https://dialnet.unirioja.es/servlet/articulo ?codigo $=5869976$

12.- Christiana F., Mpundu M., Lungowe S., Y Michelo Ch.; Seroprevalencia y determinantes de la infección por toxoplasmosis en el Hospital universitario de Lusaka. Zambia. PubMed. [Internet] 2017, [fecha de acceso 10 de noviembre 2017]; 22 Disponible en www.reibci.org/publicados/2018/abr/28 00104.pdf.
13.- Cuellar B. y Molina L. Caracterización de la Toxoplasmosis Gestacional en una Clínica Privada de Neiva (Colombia), [internet] 2014. [Fecha de acceso 20 de enero 2018]; Disponible en: https://contenidos.usco.edu.co/salud/ima ges/documentos/grados/T.G.Epidemiologi a/79.T.G-Beatriz-Helena-Cuellar-UrriagoLina-Maria-Molina-Sanchez-2014.pdf

14.- Aguayo A., Prevalencia de Toxoplasmosis y Factores de Riesgo asociados en embarazadas que acuden al primer control prenatal en el Centro de Salud de Quero, Provincia Tungurahua. Repositorio [internet] 2014 [fecha de acceso 15 de noviembre 2017]; Disponible en:

http://repositorio.uta.edu.ec/handle/123 $456789 / 4388$

15.- Fernández J, Aguiar B. y Borges I. Seroepidemiología de Toxoplasmosis en habitantes de El Viñedo, Maracay, Estado Aragua. Comunidad y Salud. Año 2015, [fecha de acceso 1 de abril 2018]; vol. 13 no 1 ene-jun. [Internet] p. 22-28.Disponible en:

http://www.scielo.org.ve/scielo.php?scrip $\mathrm{t}=$ sci_arttext\&pid=S169032932015000100004 